BROWN-HET-1255

\title{
Solving the Hierarchy Problem with Brane Crystals
}

\author{
Steven Corley and David A. Lowe \\ Department of Physics \\ Brown University \\ Providence, RI 02912, USA \\ scorley, lowe@het.brown.edu
}

\begin{abstract}
The brane world scenario advocated by Arkani-Hamed et al. transmutes the hierarchy problem into explaining why extra dimensions have sizes much larger than the fundamental scale. In this paper we discuss possible solutions to this problem by considering the compactified dimensions to be populated by a large number of branes in a crystal lattice. The experimental consequences of this scenario are described, including the presence of large energy gaps in the spectrum of Kaluza-Klein modes.
\end{abstract}

January, 2001 


\section{Introduction}

Around two years ago [1] pointed out that it was consistent with known experiments for extra dimensions to exist with sizes of order a millimeter. The motivation for this observation came from string theory, where additional "curled up" dimensions are required for the consistency of the theory. String theory also allows for the existence of D-branes, which give a way to restrict the Standard Model fields to a three dimensional slice of the higher dimensional space. Without this additional entrapment of the Standard Model fields to a brane, large extra dimensions would be in immediate contradiction with atomic physics.

The hierarchy problem becomes re-expressed as explaining why the size of the extra dimensions $r_{0}$ is much larger than the fundamental length scale. Denoting the fundamental scale by $M_{*}$ (which we can take to be of order $1 \mathrm{TeV}$ ), and the four-dimensional Planck scale $M_{P l}=10^{19} \mathrm{GeV}$, one finds

$$
M_{P l}^{2}=r_{0}^{n} M_{*}^{n+2},
$$

for $n$ flat extra dimensions with size $r_{0}$. For $n=1, r_{0}$ is required to be of cosmological scales, which is immediately ruled out. The $n=2$ case requires $r_{0}$ of order one millimeter. There remains a hierarchy between $r_{0}$ and $1 / M_{*} \approx 10^{-19} \mathrm{~m}$.

Current rounds of experiments [2], [3] are beginning to place direct constraints on the simplest scenarios. Short-range gravity experiments now probe down to $200 \mu \mathrm{m}$ strongly constraining the $n=2$ case. Accelerator experiments constrain $M_{*} \gtrsim 1 \mathrm{TeV}$ [3]. The strongest constraints arise from astrophysical considerations. Production of Kaluza-Klein modes in supernova SN 1987A places a bound $M_{*} \gtrsim 30 \mathrm{TeV}$ for $n=2$ [1].

One of the main theoretical challenges in implementing some of the proposed large extra dimension scenario's is how to stabilize the size of the extra dimensions, without introducing additional fine tuning problems. This problem should be more readily addressed in the large extra dimension scenario, versus the traditional approach of compactification at the fundamental scale, because the analysis may be carried out at the classical level. A variety of solutions have been proposed [4 9] in the warped compactification scenarios, where by warped we mean not only the Randall-Sundrum warped metric scenarios [10 13] but also cases where some other field, eg., a scalar as in [8], has a non-trivial profile in the extra dimensions. 
Another proposed solution in the unwarped compactification context of [1] is to have a large number of branes $N$ (one of which would be our world) interacting in such a way that they form a crystal lattice in the internal dimensions [14]. The interbrane separation could be the size of the fundamental length scale, but the size of the extra dimensions would nevertheless be large enough to solve the hierarchy problem for a large enough number of branes. While it is still not clear how to realize such a brane lattice crystal from a more fundamental theory such as string theory, these models are nevertheless of interest from a phenomenological point of view, especially in light of the fact that they could be tested experimentally in the near future.

One is still left with the problem of explaining the large integer $N$. We take the point of view that replacing a fine tuned continuous parameter by a large integer parameter is an improvement, as one can set the integer $N$ once and for all using initial conditions, and under suitable circumstances, this integer will be stable with respect to time evolution. Since the hierarchy is set by a conserved number $N$, it is automatically stable with respect to radiative corrections.

In the following we begin by discussing the approach to brane crystals of ArkaniHamed et al. [14]. They propose a number of scenarios for stabilizing the extra dimensions. One particularly compelling example does this without introducing additional fine tuning, aside from the usual problem with the four-dimensional cosmological constant. A problem with this scenario is the presence of unbalanced charge on a compact space. Balancing the charge leads us to consider a scenario where neutral non-BPS branes interact via a nearest neighbor potential. We show this does lead to a natural solution of the hierarchy problem. The crystal potential leads to a distinctive experimental signature for this scenario - the existence of large energy gaps in the Kaluza-Klein spectrum. Such energy gaps have also been noticed in the Randall-Sundrum scenario brane lattices discussed in [15, 16].

\section{Brane Crystal Review}

We begin by reviewing the model considered in [14]. They consider a 3-brane embedded in a universe with 3 large spatial dimensions and $n$ small spatial dimensions. The system is described by a bulk action

$$
S_{\text {bulk }}=-\int d^{4+n} x \sqrt{-\operatorname{det} g_{4+n}}\left(M_{*}^{2+n} \mathcal{R}+\Lambda-\mathcal{L}_{\text {matter }}+\cdots\right)
$$


and a brane action

$$
S_{\text {brane }}=-\int d^{4} x \sqrt{-\operatorname{det} g_{4}}\left(f^{4}+\cdots\right),
$$

where $\mathcal{L}_{\text {matter }}$ is the Lagrangian of the bulk matter fields and the ellipses denote higher derivative terms which may be dropped at low enough energies. Here $g_{4}$ denotes the induced metric on the brane, $g_{4+n}$ denotes the bulk metric, $\Lambda$ is the bulk cosmological constant and $f^{4}$ is the brane tension. Interaction terms between the branes are not included. In the next section we discuss scenarios where such interaction terms are relevant.

The metric is assumed to take the form

$$
d s^{2}=\left(\frac{r_{0}}{r}\right)^{n}\left(d t^{2}-R^{2} g_{i j} d x^{i} d x^{j}\right)-r^{2} g_{I J} d x^{I} d x^{J}
$$

where $R=R(t)$ is the scale factor of the three large dimensions and $r=r(t)$ that of the $n$ small dimensions, with $r_{0}=r(0)$. 1. Also we use lower case Latin indices $i, j, \cdots$ to denote the 3 large spatial dimensions and upper case Latin indices $I, J, \cdots$ for the $n$ small spatial dimensions.

Inserting this form of the metric into the bulk (2.1), and brane (2.2) actions and integrating over the spatial coordinates results in

$$
S=\int d t R^{3}\left(M_{*}^{n+2} r_{0}^{n}\left(-6\left(\frac{\dot{R}}{R}\right)^{2}+\frac{n(n+2)}{2}\left(\frac{\dot{r}}{r}\right)^{2}\right)-\left(\frac{r_{0}}{r}\right)^{2 n} V_{t o t}(r)\right),
$$

after an integration by parts. In obtaining this result we have assumed that $\int d^{3} x \sqrt{\operatorname{det} g_{I J}}=$ 1 and $\int d^{n} x \sqrt{\operatorname{det} g_{i j}}=1$. The potential $V_{t o t}(r)$ is given by

$$
V_{\text {tot }}(r)=\Lambda r^{n}-\kappa n(n-1) M_{*}^{n+2} r^{n-2}+f^{4}
$$

where the term proportional to $\kappa$ arises from the curvature of the $n$ dimensional space. For an $n$-sphere $\kappa=1$ and for an $n$-torus $\kappa=0$. A similar term could be added for the three large spatial dimensions, but for large $R$ would be negligible so we drop it.

For static solutions the equations of motion are simply given by

$$
V_{\text {tot }}\left(r_{0}\right)=0, \quad V_{\text {tot }}^{\prime}\left(r_{0}\right)=0
$$

1 [14] do not include the conformal factor $r^{-n}$ multiplying the large dimensions. We find it more convenient however to include it as it will lead to diagonalized kinetic terms for $r$ and $R$ in the action below and is the ordinary conformal factor appearing in Kaluza-Klein reductions. 
assuming that $R(t)=R_{0}$ is constant. Note that with the potential (2.5) $r_{0}$ is fixed entirely in terms of the bulk interaction. For the above potential (2.5) these equations constrain $\Lambda$ assuming that $r_{0}$ is chosen to solve the hierarchy problem (1.1). Specifically let's assume that the brane tension is set by the higher dimensional fundamental scale $M_{*}$ so that $f^{4} \approx M_{*}^{4}$. From (2.6), assuming the internal space is an n-sphere, it follows that

$$
\Lambda \approx \frac{M_{*}^{n+2}}{r_{0}^{2}} \approx M_{*}^{n+4}\left(\frac{M_{*}}{M_{P l}}\right)^{4 / n}
$$

where we have used (1.1).

For $N$ branes with equal tensions, the $f^{4}$ term in (2.5) is replaced by $N f^{4}$. Solving the $V_{t o t}\left(r_{0}\right)=0$ equation leads to

$$
N \approx\left(\frac{M_{P l}}{M_{*}}\right)^{2(n-2) / n}
$$

This varies from 1 for $n=2$ to $10^{20}$ for $n=6$. Note one is still left with an extra fine tuning problem, in order that the bulk cosmological constant satisfy the relation (2.7). Also note the brane number $N$ plays no role in fixing the size of the extra dimensions - this is entirely determined by fine tuning $\Lambda . N$ is fixed only by requiring the four dimensional cosmological constant vanish.

For a single brane, stability of this solution follows from the condition $V_{t o t}^{\prime \prime}\left(r_{0}\right)>0$ where $r_{0}$ solves the equations of motion (2.6). This is straightforward to see by expanding the action to quadratic order in the small perturbation $\delta r$ where $r=r_{0}+\delta r$ and then rescaling $\delta r$ so that the kinetic term is canonically normalized. The mass can then be read from the action and is given by

$$
m_{r}^{2}=\frac{1}{n(n+2)} \frac{r_{0}^{2} V_{t o t}^{\prime \prime}\left(r_{0}\right)}{M_{P l}^{2}} .
$$

Evaluating this for (2.5) gives $m_{r} \approx 1 / r_{0}$. Note the static equations of motion (2.6) and stability condition do not depend on the specific form of $V_{t o t}(r)$, and yield strong constraints on the parameters of more general potentials.

This analysis presumes that derivative couplings of the radion to higher spin KaluzaKlein modes may be neglected, which is not true in general. However this should not change the qualitative conclusions. The analysis also neglects the Hamiltonian constraint, which would take the form

$$
M_{*}^{n+2} r_{0}^{n}\left(-6\left(\frac{\dot{R}}{R}\right)^{2}+\frac{n(n+2)}{2}\left(\frac{\dot{r}}{r}\right)^{2}\right)+\left(\frac{r_{0}}{r}\right)^{2 n} V_{t o t}(r)=0 .
$$


This tells us if we really considered perturbations independent of the three noncompact spatial dimensions, we would generate an non-zero energy density everywhere in space, which would lead to expansion or contraction of $R$. This is easily remedied by generalizing to perturbations localized in the noncompact spatial dimensions.

\section{Interbrane Forces}

We now consider in more detail the effect of an interbrane potential on the above analysis. We continue to work in an approximation where the compactification is not warped, i.e. the $4+n$-dimensional metric does not depend on the internal coordinates $x^{I}$. This presumes the brane separation will be stabilized at a size parametrically larger than the fundamental length scale $1 / M_{*}$, so that treating gravity at the classical level is sufficient. We also continue to treat perturbations homogeneous in the spatial directions, with only time dependence, with the metric ansatz (2.3).

Let us generalize the brane action to $N$ branes, with a Born-Infeld type action

$S_{\text {brane }}=-\sum_{k=1}^{N} \int d^{4} x \sqrt{\operatorname{det}\left(g_{\mu \nu}-g_{I J} \partial_{\mu} X_{(k)}^{I} \partial_{\nu} X_{(k)}^{J}\right)}\left(f_{k}^{4}+\sum_{l<k} V_{\text {brane }}\left(r\left|X^{(l)}-X^{(k)}\right|\right)\right)$,

with an interbrane potential $V_{\text {brane }}$. Here we have assumed the metric is $g=g(t)$ induced on each brane is the same, and we use $X_{(k)}=X_{(k)}(t)$ to denote worldvolume fields corresponding to the brane positions in the transverse space. Indices $\mu, \nu$ label the coordinates $\left(t, x^{i}\right)$. The brane potential has been chosen to depend on the proper distance separating the branes. This leads to the factor of $r$ in potential term. In writing the Born-Infeld action we have used the worldvolume diffeomorphism invariance to fix the gauge $X_{(k)}^{\mu}=x^{\mu}$. Note that had we included spatial dependence of the fields, the brane potential would take a complicated non-local form, that is difficult to write down explicitly. A useful analogy for the branes interacting with the bulk gravitational field is gravitational waves interacting with a resonant gravitational wave detector, [17, as we will comment further below.

A natural candidate for $V_{\text {brane }}$ is a simple Coulomb coupling. This leads to the most interesting brane crystal scenario studied in [14 with "non-extensive bulk cosmological constant", where the hierarchy problem was solved without the additional fine tuning associated with the bulk cosmological constant. The radion was stabilized in the infra-red using a negative curvature term in the internal space, and the Coulomb force was used to provide a short distance stabilizing force. The difficulty with this picture is that a 
collection of like charged branes on a compact space carries infinite vacuum energy, since the electric flux has nowhere to end.

To remedy this, one could consider brane configurations with zero charge per unit cell. Of course, once branes with opposite sign are present there will be attractive forces. For supersymmetric D-branes in string theory, oppositely charged branes will annihilate. Furthermore, to our knowledge, there are no known neutral and stable branes.

The description of D-brane charges as living in K-theory groups [18] however predicts the existence of stable non-BPS branes which carry charge in a finite, or torsion, group. This charge is not associated with a gauge symmetry and there is therefore no Gauss law preventing us from considering $N$ such branes on a compact space. It remains an open question as to whether such branes could be used to construct a stable lattice configuration.

For the moment we take a phenomenological point of view and assume that a stable lattice can be constructed. These objects will then not experience a Coulomb interaction. A Van der Waals interaction is one natural interaction between such objects, arising from the interaction of induced electric dipole moments, falling off like $1 / r^{2 n}$. To obtain a stabilizing potential, this must be combined with a hard-core repulsive interaction. Taking our motivation from molecular crystals, a possible potential would be the $n$-dimensional version of the Lennard-Jones potential

$$
V_{\text {brane }}(r X)=M_{*}^{4} v\left(M_{*} r X\right), \quad v(x)=\left(\frac{\beta}{x}\right)^{4 n}-\left(\frac{\gamma}{x}\right)^{2 n}
$$

where $\beta$ and $\gamma$ are both $\mathcal{O}(1)$. One could also imagine an ionic lattice of branes, with screened Coulomb interactions. An importance difference with the Coulomb force example is that now $V_{\text {brane }}$ will scale like the number of branes $N$, rather than $N^{2}$ since nearest neighbor interactions will be dominant. The precise form of the potential will not be important for what follows.

We now want to show that the size of the internal space is fixed in terms of the number of branes $N$, rather than by using the bulk quantities $\kappa$ and $\Lambda$, which generally introduce extra fine tuning problems. We shall therefore set $\kappa$ and $\Lambda$ to zero, the former implying that the compact extra dimensions are flat and for simplicity we take their geometry to be the torus $\left(S_{1}\right)^{n}$.

Consider an interbrane potential of the form

$$
V_{\text {brane }}\left(r\left|X^{(k)}-X^{(l)}\right|\right)=M_{*}^{4} v\left(\left|X^{(k)}-X^{(l)}\right| r M_{*}\right)
$$


where $v(x)$ is not fine tuned. The fundamental scale $M_{*}$ sets the scale of the interaction. We assume $v(x)$ is short ranged, so only nearest neighbor interactions are dominant. The static equations of motion are then roughly given by $v\left(x_{0}\right)=\mathcal{O}(1)$ and $v^{\prime}\left(x_{0}\right)=0$. These have solutions $x_{0}=\mathcal{O}(1)$, or more explicitly

$$
\Delta X \approx \frac{\alpha}{r M_{*}}
$$

where $\Delta X$ denotes the coordinate separation between nearest neighbor branes and $\alpha$ is a constant of order 10 or so, which we will discuss momentarily. Using the fact that the coordinate periodicity around any of the $S^{1}$ 's of our extra dimensions is 1 and summing up the $\Delta X$ 's along one of these dimensions yields the static value of $r$,

$$
r_{0} \approx \alpha \frac{N^{1 / n}}{M_{*}}
$$

We find therefore that the size of the internal dimensions is set by the number of branes along with the fundamental scale. The value of $r_{0}$ however was already fixed in (1.1) and thus yields the necessary number of branes

$$
N \approx \frac{1}{\alpha^{n}}\left(\frac{M_{P l}}{M_{*}}\right)^{2}=\frac{1}{\alpha^{n}} 10^{32} .
$$

$\alpha=1$ corresponds to one brane per fundamental volume in the internal space, saturating the number of branes. We have therefore required that $\alpha$ is of order 10 or larger (but not so large that we have another fine tuning problem) in order that classical gravity be a good approximation.

The effective potential induced for $r$ takes the form

$$
V_{\text {tot }}(r)=N f^{4}+N V_{\text {brane }}\left(r / N^{1 / n}\right)
$$

where integrating out the brane coordinates sets the coordinate distance between neighboring branes to $1 / N^{1 / n}$. Vanishing of the four-dimensional cosmological constant requires one fine tuning, corresponding to $V_{t o t}=0$, but note no additional fine tuning is needed. The mass of the radion may be obtained using (2.9), which gives

$$
m_{r}=M_{*} / \alpha^{n / 2}
$$

We have so far taken vanishing bulk cosmological constant. This is expected at treelevel if supersymmetry is unbroken in the bulk. However if supersymmetry is broken on 
the branes then a cosmological constant will appear at one loop and could change the results. If the breaking takes place at the fundamental scale $M_{*}$ on the branes then the induced mass splittings in the bulk are given by a tree-level gravitational effect [14]

$$
\Delta m^{2} \approx N \frac{M_{*}^{4}}{M_{P l}^{2}}=\frac{M_{*}^{2}}{\alpha^{n}},
$$

where the last expression was obtained by evaluating $r_{0}$ at (3.5). By dimensional analysis, this induces a cosmological constant $\Lambda_{\text {quantum }} \approx\left(\Delta m^{2}\right)^{(4+n) / 2}$ so that the potential gets a contribution of

$$
V_{\text {quantum }}(r) \approx \frac{M_{*}^{4+n}}{\alpha^{(4+n) n / 2}} r^{n} \rightarrow M_{*}^{4} N\left(\frac{x_{0}}{\alpha^{(4+n) / 2}}\right)^{n}
$$

where $x_{0}$ was defined above. Therefore at $x_{0} \approx \alpha$ the induced cosmological constant contribution to the potential is subdominant and our original estimates above still apply.

We expect the brane crystal scenario will only work when the number of extra dimensions $n \geq 3$. This follows from [19] where it is shown that classical crystal lattices do not exhibit long-range order in dimensions two or less. For $M_{*} \approx 1 \mathrm{TeV}$, (1.1) places $r_{0} \approx 10^{-7} \mathrm{~m}$ for $n=3$.

\section{Experimental Consequences}

To understand the experimental consequences of our scenario we must investigate the spectrum of the theory as well as the couplings to Standard Model fields. To do this it is convenient to first fix the $(4+n)$ dimensional diffeomorphism invariance. For linearized perturbations $h_{M N}$ about a flat metric $\tilde{g}_{M N}$ (with $M, N$ labeling the $4+n$-dimensional space) an infinitesimal diffeomorphism generated by a vector $\xi_{M}$ acts on the metric as

$$
h_{M N} \rightarrow h_{M N}+\nabla_{M} \xi_{N}+\nabla_{N} \xi_{M}
$$

where we have decomposed the metric into a background part $\tilde{g}_{M N}$ and a fluctuation $h_{M N}$ as

$$
g_{M N}=\tilde{g}_{M N}+h_{M N}
$$

On the $X_{(k)}^{I}$ 's this will act as

$$
X^{I} \rightarrow X^{I}-\xi^{I}
$$


Part of the gauge symmetry can be fixed by demanding

$$
\partial^{M} \bar{h}_{M N}=0,
$$

where

$$
\bar{h}_{M N}=h_{M N}-\frac{1}{2} \tilde{g}_{M N} h,
$$

and indices are being raised and lowered with the background metric. This gauge choice does not fix the diffeomorphism invariance corresponding to vectors satisfying the $(4+n)$ dimensional wave equation $\partial^{M} \partial_{M} \xi_{N}=0$.

We begin by expanding the Born-Infeld piece of the brane action for a general perturbation

$$
\begin{aligned}
\int d^{4} x \sqrt{\operatorname{det} G_{\mu \nu}} & =\int d^{4} x \sqrt{\operatorname{det} \tilde{g}_{\mu \nu}}\left(1+\frac{1}{2} \tilde{g}^{\mu \nu} n_{\mu \nu}+\right. \\
& \left.\frac{1}{8}\left(\tilde{g}^{\gamma \beta} \tilde{g}^{\mu \nu}-\tilde{g}^{\mu \gamma} \tilde{g}^{\nu \beta}-\tilde{g}^{\mu \gamma} \tilde{g}^{\nu \beta}\right) n_{\gamma \beta} n_{\mu \nu}+\cdots\right),
\end{aligned}
$$

where $G_{\mu \nu}$ is the full induced metric on the brane, and

$$
n_{\mu \nu}=h_{\mu \nu}+h_{\mu I} \frac{\partial X^{I}}{\partial x^{\nu}}+h_{I \nu} \frac{\partial X^{I}}{\partial x^{\mu}}+\tilde{g}_{I J} \frac{\partial X^{I}}{\partial x^{\mu}} \frac{\partial X^{J}}{\partial x^{\nu}} .
$$

Since $h_{\mu \nu}$ picks up a nontrivial second-order contribution under diffeomorphisms of the form $\xi^{\mu}=0, \xi^{I} \neq 0$

$$
h_{\mu \nu} \rightarrow h_{\mu \nu}+h_{\mu I} \partial_{\nu} \xi^{I}+h_{\nu I} \partial_{\mu} \xi^{I}
$$

as may be seen by expanding (4.1), it is convenient to write $h_{\mu \nu}=j_{\mu \nu}+h_{\mu I} h_{\nu}^{I}$, so $j_{\mu \nu}$ will then be invariant under such diffeomorphisms. This redefinition also makes clear that (4.6) gives rise to a mass term for the four-dimensional vector fields $h_{\mu I}$. A different approach to seeing the vector fields become massive is discussed in [1]. However since we are tuning the four-dimensional cosmological constant to zero, the overall coefficient of (4.6) will vanish, and the vectors will be massless (as is the graviton).

To examine terms arising from the brane potential, we again restrict to perturbations independent of the spatial directions. We gauge fix the fluctuations of the brane coordinates $X_{(k)}^{I}$ to zero, as explained in more detail in appendix A. At quadratic order in the fluctuations, then only the radion modes $\bar{h}_{I J}$ couple to the brane coordinates $X_{(k)}^{I}$ in the action.

The modes independent of the internal dimensions will therefore be a massless graviton $\bar{j}_{\mu \nu}$, a set of massless vector fields $\bar{h}_{\mu}^{I}$, and a set of massive radion scalars $\bar{h}_{I J}$ with mass 
given by (3.8), following through the same calculation. Each of these modes will be at the bottom of a tower of Kaluza-Klein states which are standing waves in the internal dimensions. These may be treated in the same way as Bloch waves [20]. For the low lying modes, the effects of interactions may be neglected (at least for sufficiently large $\alpha$ ). This is precisely analogous to the case of gravitational waves propagating through a resonant detector, where one needs to go to next order in the equations of motion to see the effect of gravitational interactions on the response of the detector [17]. This gives rise to a typical Kaluza-Klein spectrum for the spin-two and vector modes $m_{k}=\left|k / r_{0}\right|$, while for the radion modes $m_{k}^{2}=m_{r}^{2}+\left(k / r_{0}\right)^{2}$.

It is also interesting to calculate the energy band gap at the edge of the Brillouin zone boundary, where we have standing waves commensurate with the lattice spacing of the crystal. In general, interaction effects will become large there. For the radion modes, we can get a reasonable estimate of this band gap by taking into account only the interaction of the radion through the brane potential. For plane wave modes propagating in the $I$ 'th direction, the equations of motion are the same as that of an electron moving in a periodic array of delta function potentials. This is a special limit of the Kronig-Penney model [21]. The wavefunction takes the form

$$
r(x)=e^{i k x} u(x)
$$

where $x$ is the $x^{I}$ in question, and $u(x)$ is periodic under lattice translations. Solving the equations of motion for $u(x)$ a linear combination of $e^{ \pm i K x}$ yields

$$
\cos (k a)=\cos (K a)+\frac{1}{2 \alpha^{n-2}} \frac{\sin (K a)}{K a}
$$

where $a$ is the lattice spacing $r_{0} / N^{1 / n}$. Here $K a$ is to be identified with $\alpha m / M_{*}$, where $m$ is the mass of the mode. For the first Brillouin zone boundary $k=\pi / a$. Solving this equation yields the band gap $\Delta m^{2} \sim M_{*}^{2} / \alpha^{n}$. This is the same form as the mass gap of the radion near $k=0$.

Higher order interactions also lead to a band gap for the spin-two and vector modes at the Brillouin zone boundary. Estimating the energy difference between a standing wave with nodes on the branes versus a standing wave with peaks on the branes leads to the same calculation as in (3.9). We therefore expect the band gap $\Delta m^{2}$ to be of roughly the 


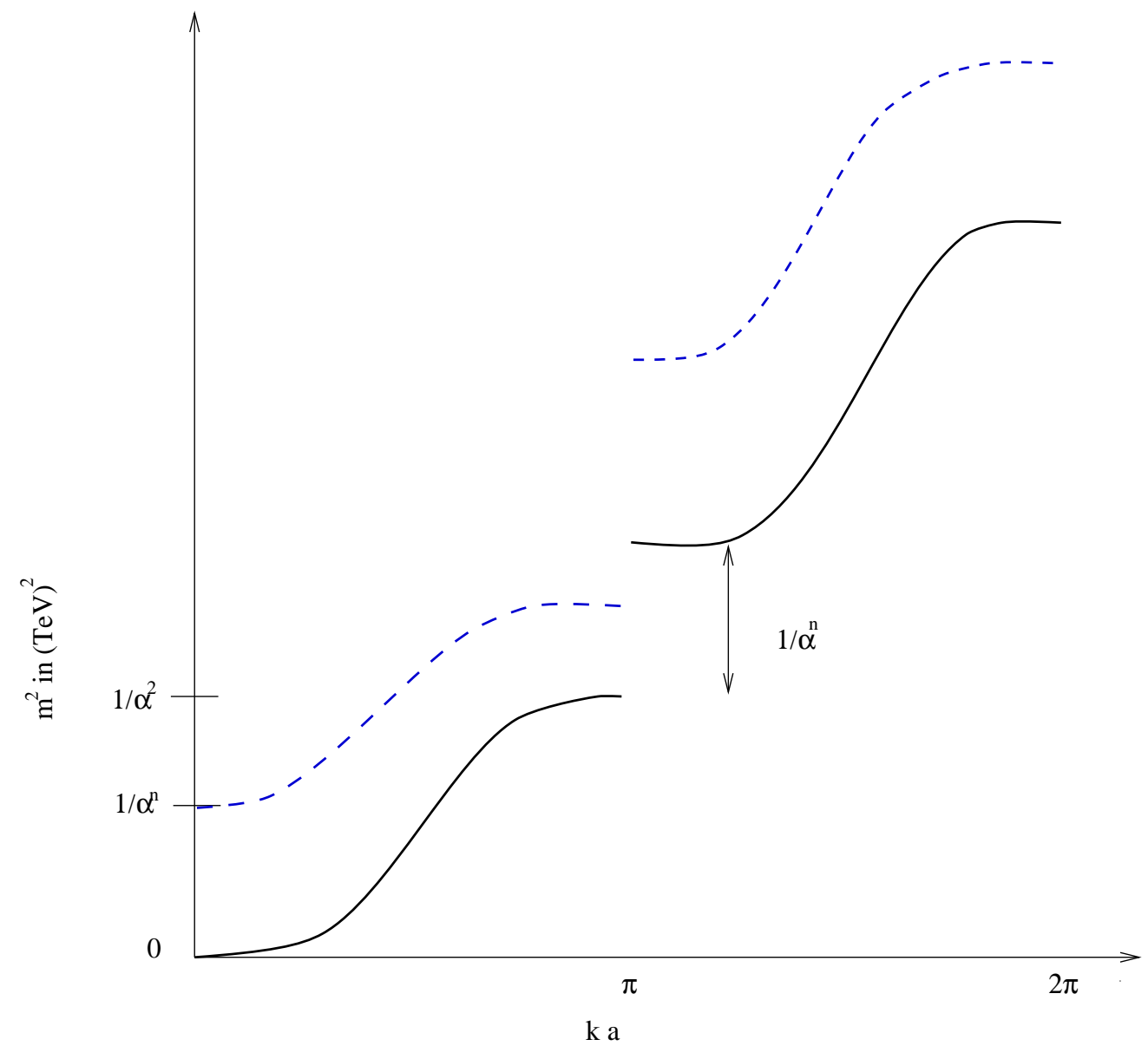

Fig. 1: Illustration of expected band structure in the spectrum of KaluzaKlein states. The mass squared is plotted versus the wavevector in the internal dimension $k$ times the lattice spacing $a \sim \alpha / M_{*}$. The orders of magnitude of the scales of the band width and band gaps are indicated.

same order of magnitude as for the radion modes. The picture of the band structure that emerges is illustrated in figure 1.

The Standard Model fields are coupled to the induced metric on the brane via the usual covariant couplings. Expanding these terms about the background metric we find that the coupling between the bulk metric fluctuations discussed above and the Standard model fields will be suppressed by $1 / M_{P l}$. The analysis of the phenomenological constraints of [1] will therefore carry over to the brane crystal model unchanged.

Acknowledgments We thank A. Houghton, R. Myers, R. Pelcovits and L. Randall for helpful discussions. This research is supported in part by DOE grant DEFE0291ER40688-Task A. 


\section{Appendix A. Gauge fixing the brane coordinates}

As noted above, the linearized Einstein equations in covariant gauge $\partial^{M} \bar{h}_{M N}$ have a residual gauge freedom associated with diffeomorphisms satisfying $\partial^{M} \partial_{M} \xi_{N}=0$. This freedom can be used to set the fluctuations of the transverse brane coordinates to zero given some assumptions. Specifically the brane coordinates transform under a diffeomorphism as

$$
X_{(k)}^{I}\left(x^{\mu}\right) \rightarrow X_{(k)}^{I}\left(x^{\mu}\right)-\xi^{I}\left(x^{\mu}, x^{J}\right),
$$

where we have assumed that $\xi^{\mu}=0$. To set the brane coordinate fluctuation to zero therefore only requires fixing $\xi^{I}$ on the brane, and it's extension off the brane can be chosen at our convenience. In this case we choose it's extension off the brane so that it solves the $(4+n)$-dimensional wave equation. In general this will not be possible. This follows by noting that the $\xi^{I}$ live in the bulk spacetime and therefore must satisfy the periodicity conditions of the extra dimensions. As a result the $\xi^{I}$ cannot have arbitrary dependence on the $x^{\mu}$ coordinates if they are to satisfy the wave equation. To be explicit consider a mode decomposition of $\xi^{I}$ where one of the modes satisfies $\partial^{\mu} \partial_{\mu} \xi^{I}=m^{2} \xi^{I}$. For $\xi^{I}$ to satisfy the $(4+n)$-dimensional wave equation we then need $\partial^{J} \partial_{J} \xi^{I}=m^{2} \xi^{I}$. For generic values of $m$ there will be no solution to this equation that satisfies the required periodicity conditions in the extra dimensions. Rather the 4-dimensional mass $m$ will be quantized according to $m=\sqrt{q_{1}^{2}+\cdots+q_{n}^{2}} / r_{0}$ for arbitrary integers $q_{i}$. There is no such constraint on the dependence of the brane coordinates on $x^{\mu}$ however because they are functions only of the $x^{\mu}$. We assume for simplicity nevertheless that the brane coordinate fluctuations can be gauged away in this manner. In other words we assume that the mode decomposition of the brane coordinates contains only fluctuations with the quantized masses given above therefore allowing us to gauge them away.

\section{Appendix B. Alternate derivation of linearized equations}

Given these gauge conditions described in appendix A, we now give a more general derivation of the linearized equations of motion describing the metric fluctuations. We could try to write down a potential term describing the interactions between the branes and then gauge fixing as described above, but this turns out to be somewhat subtle. We therefore argue using symmetry considerations. The bulk contribution comes only from the Einstein-Hilbert term in the action (2.2). In the gauge (4.4) it is well known that this 
contributes only $\partial^{P} \partial_{P} \bar{h}_{M N}$ to the linearized equations of motion. From the brane terms in the action we expect the linearized equations of motion to contain a sum of $\delta$-function terms in the extra dimensions corresponding to the fixed brane positions with coefficients determined by symmetry and dimensional analysis. Specifically we find

$$
\begin{aligned}
& \partial^{M} \partial_{M} \bar{h}_{\mu \nu}=\frac{M_{*}^{4}}{M_{P l}^{2}} \sum_{(k)}\left(a_{(k)} \bar{h}_{\mu \nu}+b_{(k)} \tilde{g}_{\mu \nu} \bar{h}_{4}\right) \delta^{(n)}\left(X^{I}-X_{(k)}^{I}\right) \\
& \partial^{M} \partial_{M} h_{\mu I}=\frac{M_{*}^{4}}{M_{P l}^{2}} \sum_{(k)} c_{(k)} h_{\mu I} \delta^{(n)}\left(X^{I}-X_{(k)}^{I}\right) \\
& \partial^{M} \partial_{M} \bar{h}_{I J}=\frac{M_{*}^{4}}{M_{P l}^{2}} \sum_{(k)}\left(d_{(k)} \bar{h}_{I J}+e_{(k)} \tilde{g}_{I J} \bar{h}_{n}\right) \delta^{(n)}\left(X^{I}-X_{(k)}^{I}\right)
\end{aligned}
$$

where $\bar{h}_{4}=\tilde{g}^{\mu \nu} \bar{h}_{\mu \nu}, \bar{h}_{n}=\tilde{g}^{I J} \bar{h}_{I J}$, and the coefficients $a_{(k)}, \ldots, e_{(k)}$ are all $\mathcal{O}(1)$. The overall factor of $M_{*}^{4} / M_{P l}^{2}$ on the right-hand-sides of all equations is easy to understand by going to a coordinate system $\tilde{X}^{I}=r_{0} X^{I}$. In these coordinates $M_{*}$ is the only dimensionful parameter so that $M_{*}^{2-n}$ would have to be the overall coefficient following from dimensional analysis. Going back to the $X^{I}$ then yields the above coefficient.

Decomposing the fluctuations $\bar{h}_{M N}$ into eigenstates of the 4-dimensional wave operator yields

$$
\partial^{M} \partial_{M} \bar{h}_{M N}=\left(-r_{0}^{-2} \partial_{I} \partial_{I}+m^{2}\right) \bar{h}_{M N}
$$

for a mode with 4-dimensional mass $m$. It is now straightforward to estimate the spectrum of metric fluctuations following the discussion around (4.9). In particular taking a plane wave ansatz for the metric fluctuations one recovers the relation (4.10) from which we find that the first excited state and mass gap energies will be or order $m^{2}, \Delta m^{2} \approx M_{*}^{2} / \alpha^{n}$ respectively.

The discussion so far applies for all three equations of motion in (B.1), so in particular it implies that the lowest energy fluctuation of $h_{\mu \nu}$ would have four-dimensional mass of order $M_{*} / \alpha^{n / 2}$. This is of course unacceptable if we are to recover Newtonian gravity on our brane. For the $h_{\mu \nu}$ equation therefore we must tune the $a_{(k)}$ and $b_{(k)}$ coefficients so that we have a massless fluctuation, or massless four-dimensional graviton. This corresponds to fine tuning the four-dimensional cosmological constant to zero. Note however that this fine tuning will not in general imply that the low lying vector fluctuations $h_{\mu I}$ or radion fluctuations $h_{I J}$ will be massless. 


\section{References}

[1] Nima Arkani-Hamed, Savas Dimopoulos and G. Dvali, Phys. Lett. B429 (1998) 263; Phys. Rev. D59 (1999) 086004; I. Antoniadis, N. Arkani-Hamed, S. Dimopoulos and G. Dvali, Phys. Lett. B436 (1998) 257.

[2] G.L. Smith, C.D. Hoyle, J.H. Gundlach, E.G. Adelberger, B.R. Heckel, and H.E. Swanson, Short-range Tests of the Equivalence Principle, Phys. Rev. D61 022001 (2000); C.D. Hoyle, U. Schmidt, B.R. Heckel, E.G. Adelberger, J.H. Gundlach, D.J. Kapner and H.E. Swanson, Submillimeter Tests Of The Gravitational Inverse Square Law: A Search For 'large' Extra Dimensions, hep-ph/0011014.

[3] G. Landsberg, Mini-Review on Extra Dimensions, hep-ex/0009038.

[4] W.D. Goldberger and M.B. Wise, Phys. Rev. Lett. 83 (1999) 4922, hep-ph/9907447.

[5] I. Antoniadis and C. Bachas, Phys. Lett. B450 (1999) 83, hep-th/9812093.

[6] N. Arkani-Hamed, S. Dimopoulos and J. March-Russell, Logarithmic unification from symmetries enhanced in the submillimeter infrared, In "The many faces of the superworld", ed. M.A. Shifman, pp. 627-648, hep-th/9908146.

[7] G. Dvali, Phys. Lett. B459 (1999) 489, hep-ph/9905204.

[8] N. Arkani-Hamed, L. Hall, D. Smith, and N. Weiner, Phys. Rev. D62 (2000) 105002, hep-ph/9912453.

[9] A.G. Cohen and D.B. Kaplan, Phys. Lett. B470 (1999) 52, hep-th/9910132.

[10] L. Randall and R. Sundrum, Phys. Rev. Lett. 83 (1999) 3370, hep-ph/9905221.

[11] L. Randall and R. Sundrum, Phys. Rev. Lett. 83 (1999) 4690, hep-ph/9906064.

[12] M. Gogberashvili, Hierarchy problem in the shell universe model, hep-ph/9812296.

[13] M. Gogberashvili, Europhys. Lett. 49 (2000) 396, hep-ph/9812365.

[14] Nima Arkani-Hamed, Savas Dimopoulos, and John March-Russell, Stabilization of Sub-Millimeter Dimensions: The New Guise of the Hierarchy Problem, hepth/9809124.

[15] S. Nam, JHEP 04 (2000) 002, hep-th/9911237.

[16] N. Kaloper, Crystal Manyfold Universes in AdS Space, Phys. Lett. B474 (2000) 269, hep-th/9912125.

[17] See for example, C.W. Misner, K.S. Thorne and J.A. Wheeler, Gravitation (Freeman, San Francisco).

[18] E. Witten, D-Branes And K-Theory, JHEP 9812 (1998) 019, hep-th/9810188.

[19] N.D. Mermin, Crystalline Order in Two Dimensions, Phys. Rev. 176 (1968) 250.

[20] See for example, C. Kittel, Introduction to Solid State Physics, John Wiley and Sons, Inc.

[21] R. de L. Kronig and W.G. Penney, Proc. Roy. Soc. (London), A130 (1931) 499. 